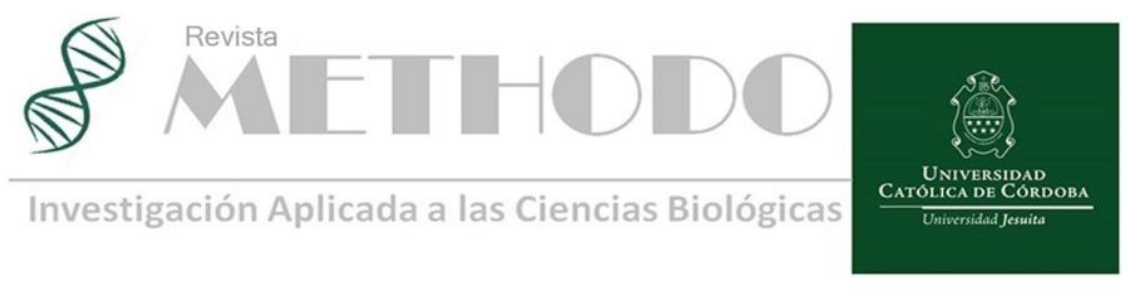

\title{
Mínimo espesor dentinario de conductos de premolares preparados para postes de fibra
}

\section{Minimum dentin thickness in premolars after root canal and fiber post preparation}

\author{
Sofía Alcaraz ${ }^{1}$, Gabriela Martin ${ }^{1}$, Georgette Arce Brison ${ }^{1}$, Sandra Arguelles $^{1}$, Catalina Solla ${ }^{1}$ \\ 1 Universidad Católica de Córdoba Facultad de Ciencias de la Salud, Carrera de Especialización de Endodoncia \\ Correspondencia: Sofía Alcaraz. Universidad Católica de Córdoba. Carrera de Endodoncia.- Córdoba, Argentina; email: so-alcaraz@hotmail.com
}

\section{Resumen}

INTRODUCCIÓN: Los elementos tratados endodónticamente pierden estructura dentinaria, como resultado de caries, preparación de acceso endodóntico y ensanchamiento para pernos. La preparación del espacio para poste consiste en conformar el conducto radicular, que posee formas irregulares y variables, generando un espacio de formas regulares que se correspondan con las del poste. El espacio para el poste no debe exceder un tercio del diámetro de la raíz y el espesor de dentina remanente no debe ser menor de $1 \mathrm{~mm}$.

OBJETIVO GENERAL: Evaluar in vitro el espesor de dentina residual en premolares con un solo conducto luego de la instrumentación endodóntica y posterior preparación para poste de fibra de vidrio.

OBJETIVOS ESPECÍFICOS: 1) Medir el espesor dentinario desde la superficie del conducto hasta la superficie externa de la raíz. 2) Reconocer la zona de mínimo espesor de dentina en el espacio del conducto preparado para poste, entre la obturación de gutapercha y el hombro tallado en el elemento dentario. 3) Comparar la ubicación de la zona de mínimo espesor entre los elementos dentarios. 4) Determinar en qué cara del elemento dentario, libre o proximal, se observa el mínimo espesor dentinario.

MATERIALES Y MÉTODOS: Se seleccionaron 20 premolares extraídos con un solo conducto radicular. Se tomaron radiografías de frente y perfil, y posteriormente se talló la cavidad de acceso coronario. Se realizó un pre-tallado coronario y se instrumentaron los conductos con el Sistema Reciproc Blue (VDW). Se procedió a la obturación con cono de gutapercha y cemento Ad-Seal (META) mediante la técnica de compactación lateral. Se realizó la preparación para poste de fibra de vidrio con fresa Gates Glidden $\mathrm{n}^{\circ} 2$, fresa Peeso $\mathrm{n}^{\circ} 1$, seguida de la $\mathrm{n}^{\circ} 2$ y se finalizó con el ensanchador para postes RTD $\mathrm{N}^{\circ} 2$; dejando $5 \mathrm{~mm}$ de obturación con gutapercha a nivel apical. Se tomó una tomografía computada de haz cónico (CBCT) sobre la cual se realizaron las mediciones de los espesores de dentina, desde la superficie del conducto radicular hasta la superficie externa de la raíz, en las caras libres y proximales del elemento dentario. Se registraron las mediciones a la altura del hombro de tallado coronario, a la altura del conducto donde comienza la obturación con gutapercha y en un punto equidistante entre el hombro y la gutapercha. Los datos obtenidos fueron registrados en una hoja de captura de datos y analizados estadísticamente mediante el test Student Newman Keuls.

RESULTADOS: En 5 piezas dentarias el espesor residual de dentina fue menor a $1 \mathrm{~mm}$. El promedio de mesial y distal fue significativamente menor que las caras libres. Los mínimos espesores dentinarios se presentaron en mesial y distal del nivel apical, seguido por mesial de la zona media.

CONCLUSIÓN: La preparación del conducto para la colocación de postes de fibra en premolares implica remoción de dentina y el mínimo espesor remanente fue observado en las caras mesial y distal de la raíz.

Revista Methodo: Investigación Aplicada a las Ciencias Biológicas. Universidad Católica de Córdoba. Jacinto Ríos 571 Bo Gral. Paz. X5004FXS. Córdoba. Argentina. Tel.: (54) 3514517299 / Correo: methodo@ucc.edu.ar / Web: methodo.ucc.edu.ar | ARTICULO ORIGINAL Methodo 2019;4(4):115-121 
Palabras claves: premolares, dentina, Tomografía Computada Cone- Beam, poste de fibra.

\begin{abstract}
INTRODUCTION: Endodontically treated teeth lose dental structure, as a result of caries, endodontic access and post space preparation. This space is prepared by conforming the root canal which has irregular and variable shapes, creating a space which shape corresponds with the posts.Post space should not exceed one third of the root diameter and the residual dentin thickness should not be less than one millimiter GENERAL OBJECTIVE: To evaluate in vitro the residual dentin thickness of premolar root canals after endodontic instrumentation and post space preparation.

SPECIFIC OBJECTIVES: 1 . To measure dentin thickness from the canal to the external surface of the root. 2. To recognize the area with mínimum dentin thickness in the post space. 3. To compare the location of the area with minimum dentin thickness between theeth. 4.To determine the site of the tooth where the minimum dentin thickness is located.

MATERIAL AND METHODS: Twenty extacted premolars with one canal were selected. Buccal and proximal radiographs were taken and access cavities were prepeared. A pre-carved was done in the crown, and the canals were instrumented with the Reciproc Blue system (VDW). Root canals were obturated with gutapercha cones and AD SEAL sealer, using lateral condensation technique. Post space preparation was performed No 1 Gates-Glidden drills, No 1 and No 2 Peesso drills and No 2 specific RTD drills, leaving five millimeters of gutapecha obturation in the apical level. A Cone- Beam Computed Tomography (CBCT) was taken and cross-sections measurements were calculated from the canal to the external surface of the root. Residual dentin thickness was registered at three levels: obturation with gutapecha, the carved shoulder and equidistant point between previous both points. Obtained data was registered for statistical analysis by Student Newman Keuls test.

RESULTS: Residual dentin thickness was lower than $1 \mathrm{~mm}$ in 5 teeth. The difference between mesial/distal dentin thickness and buccal/lingual dentine thicknes was statistically significant. Minimum dentin thickness were observed at mesial and distal sides, at apical level, followed by medium level.

CONCLUSION: Root canal and fiber post preparation in premolars remove dentin structure and the minimum residual thickness was observed at mesial and distal sides of the root.
\end{abstract}

Keywords: premolar, dentin, Cone-Beam Computed Tomography, Post

\section{Introducción}

Los elementos tratados endodónticamente, comúnmente, pierden considerables cantidades de estructura dentaria, como resultado de caries, restauraciones previas, trauma, fracturas y preparación de acceso para el tratamiento de conducto $^{1}$. El potencial de fractura de los elementos tratados endodónticamente aumenta proporcionalmente con la cantidad de estructura dentaria removida ${ }^{2}$. Las fracturas radiculares verticales pueden ser causadas por procedimientos restauradores, cómo la preparación del conducto para poste o la selección incorrecta del mismo ${ }^{3}$. En los últimos años el uso de postes, se ha convertido en un procedimiento de restauración común ${ }^{4}$. Los postes de base orgánica reforzados con fibras están compuestos por una matriz de resina que mantiene cohesionadas fibras, las cuales actúan como refuerzo estructural ${ }^{5}$. Cumplen funciones específicas en la rehabilitación post-endodóntica como conectar la porción coronaria con la radicular cuando los tejidos coronarios remanentes son escasos y/o poco resistentes, y estabilizar mecánicamente la porción coronaria del diente actuando como un alma rígida ${ }^{6,7}$.

La preparación del espacio para poste consiste en conformar el conducto radicular, que posee formas irregulares y variables, generando un espacio de formas regulares que se correspondan con las del poste $^{8}$. Este espacio es creado a través de la remoción de la gutapercha. Aunque varias técnicas para restauraciones con postes han sido propuestas en la literatura, todas requieren una preparación adicional del conducto para una adaptación óptima ${ }^{9}$. La técnica más común y rápida consiste en el uso de instrumental rotatorio ${ }^{10}$. Durante esta preparación, el operador debe conocer la anatomía interna específica de cada raíz a tratar ${ }^{11}$.

Los premolares que presentan un solo conducto son más anchos en sentido vestíbulo-palatino que en sentido mesio-distal ${ }^{12}$. Presentan depresiones por mesial y distal, con una forma oval en su corte transversal, que se va adelgazando desde la cámara hacia el ápice ${ }^{13}$. Siguiendo los parámetros clínicos pre establecidos, el espacio para el poste no debe exceder un tercio del diámetro de la raíz ${ }^{14,15}$ y el 
espesor de dentina remanente no debe ser menor de 1 milímetro desde la pared del conducto hasta la pared externa de la raíz ${ }^{16,17}$.

En los últimos años, se ha introducido en el mercado Reciproc Blue (VDW), un sistema de instrumentación mecanizada para ser usado con movimientos reciprocantes. Está fabricado con una aleación de niquel titanio, a la cual se le aplican tratamientos térmicos para mejorar la resistencia a la fatiga cíclica y la flexibilidad ${ }^{18}$. Presenta una sección transversal en forma de "s" con una punta no cortante y dos bordes cortantes ${ }^{19}$. El instrumento color rojo tiene un calibre \#25 y conicidad 0.8 .

Los postes de fibra Macro Lock (RTD) están compuestos por fibras de cuarzo y resina epóxica. Son altamente radiopacos. Se presentan en cuatro medidas: 0,8 / 1.35 (amarillo), 0,8/1.45 (rojo), 1/1.65 (azul) y 1/1.85 (verde). Cada uno de los postes se presenta con su respectivo taladro para ensanchar el conducto ${ }^{20}$.

En otro aspecto, Souza et $\mathrm{al}^{21}$ ha demostrado que las radiografías periapicales sobre estiman el espesor de dentina residual de las paredes proximales. La Tomografía Computada de Haz Cónico (CBCT) es un método de diagnóstico certero $^{22}$ y ofrece imágenes de los elementos dentarios en alta resolución superando a las radiografías en dos dimensiones, que no brindan información suficiente ${ }^{23}$. Tiene una serie de líneas de detectores múltiples, que permiten tomar múltiples cortes simultáneamente, con tiempos más rápidos y menor exposición a la radiación ${ }^{24}$. $\mathrm{El}$ volumen de imágenes adquiridas por una CBCT está formado por vóxeles. Un vóxel es un pixel 3D. Debido a que los datos se capturan en un volumen, todos los voxeles son isotrópicos y permiten medir con exactitud los elementos dentarios en diferentes direcciones ${ }^{25}$.

\section{Objetivo}

GENERAL

Evaluar in vitro el espesor de dentina residual en premolares con un solo

conducto luego de la instrumentación endodóntica y posterior preparación

para poste de fibra de vidrio.

ESPECÍFICOS

1. Medir el espesor dentinario desde la superficie del conducto hasta la

superficie externa de la raíz

2. Determinar cuál es el valor del mínimo espesor dentinario.

3. Reconocer la zona de mínimo espesor de dentina en el espacio del

conducto preparado para poste, entre la obturación de gutapercha y el hombro tallado en el elemento dentario.

4. Comparar la ubicación de la zona de mínimo espesor entre los

elementos dentarios.

5. Determinar en qué cara del elemento dentario, libre o proximal, se

observa el mínimo espesor dentinario.

\section{Material y métodos}

Se estudiaron premolares extraídos de pacientes que concurrieron a la Cátedra de Cirugía de la Clínica Odontológica Sixto Castellano de la Universidad Católica de Córdoba, quienes firmaron el correspondiente consentimiento informado. Los premolares fueron extraídos por razones ajenas a este estudio.

Tamaño de muestra: 20 elementos dentarios

Criterios de inclusión: presentar un solo conducto radicular con ápices cerrados.

Criterios de exclusión: presencia de caries, anomalías, restauraciones y obturaciones

Se seleccionaron 20 premolares extraídos con un solo conducto radicular, con ápices cerrados, libres de caries, anomalías, restauraciones y obturaciones. Se eliminó todo resto de tejido orgánico y se tomaron radiografías de frente y perfil para verificar la anatomía de los conductos radiculares.

Con fresa redonda $\mathrm{N}^{\circ} 4$ se realizó cavidad de acceso coronario y rectificación de las paredes con fresa Endo Z (Dentsply Maillefer).

Posteriormente, se realizó un pre-tallado coronario de los elementos dentarios, con fresa cilíndrica, dejando el hombro de la preparación a nivel

de la unión cemento-esmalte.

Se realizó el cateterismo de los conductos con lima K \#15 y se determinó la conductometría, introduciendo la lima K \# 15 en el conducto hasta visualizarla en el foramen. A esta medida se le restó $1 \mathrm{~mm}$ y el resultado obtenido fue la Longitud de Trabajo (LT). Se instrumentaron los conductos con técnica mecanizada, con el Sistema Reciproc Blue (VDW), específicamente con la lima color rojo calibre 25/08. Se llegó a la longitud de trabajo en tres tiempos, trabajando por tercios, irrigando con hipoclorito de sodio al $2,5 \%$ y manteniendo la permeabilidad con lima $\mathrm{K}$ \# 15 entre cada instrumento rotatorio. Se secaron los conductos con conos de papel y se procedió a la obturación con cono de gutapercha 25/06 (META), conos accesorios y cemento Ad-Seal (META) mediante la técnica de compactación lateral.

Una vez finalizado el tratamiento endodóntico, se procedió a agrupar los dientes en cuatro grupos, de cinco cada uno y montarlos en bloques de silicona por condensación (Speedex, Coltene). Se tomó una Tomografía Computada Cone Beam (CBCT) con 
vóxeles isotrópicos de 90 um, en una adquisición con $80 \mathrm{KV}$ y $5 \mathrm{~mA}$.

Se realizó la preparación para poste de fibra de vidrio siguiendo el protocolo para postes RTD. Se usó la siguiente secuencia de instrumentos:

-Fresa Gates Glidden n² a 1000 rpm

-Fresa PEESO n ${ }^{\circ} 1$ a 1000 rpm

-Fresa PEESO n² a 1000 rpm

-Ensanchador o "taladro" específico de la marca de postes RTD N², color rojo, 90.06

Con cada uno de los instrumentos se trabajó hasta una longitud pre establecida de acuerdo a la longitud del conducto, dejando $5 \mathrm{~mm}$ de obturación con gutapercha a nivel apical. Finalizada esta instancia se tomó una segunda CBCT con la misma metodología y aparatología anteriormente mencionada. Sobre la segunda CBCT se realizaron las mediciones de los espesores de dentina, desde la superficie del conducto radicular hasta la superficie externa de la raíz, en las caras libres y proximales (mesial, distal, vestibular y palatino) del elemento dentario. $\mathrm{Se}$ registraron las mediciones a tres alturas diferentes de la raíz:

1- Punto coronario: hombro de tallado coronario 2- Punto gutapercha: altura del conducto donde comienza la obturación con gutapercha

3- Punto medio: altura equidistante entre el punto coronario y el punto gutapercha. (Figura 1)
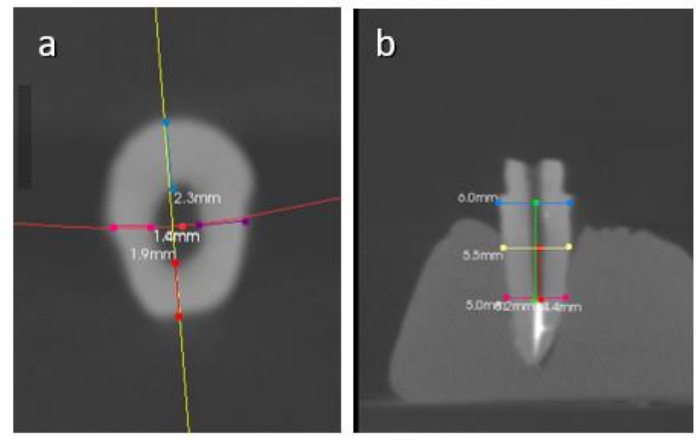

Figura 1. Tomografía computada Cone-Beam. a) Mediciones de los espesores de dentina desde la superficie del conducto radicular hasta la superficie externa de la raíz, en las caras libres y proximales: mesial, distal, vestibular y palatino. b) A tres niveles de la raíz: punto coronario, punto apical donde comienza la obturación con gutapercha y punto medio equidistante entre los anteriores.

\section{Variables}

Variables dependientes: mínimo espesor dentinario

Variables independientes: preparación para poste de fibra.

\section{Análisis estadísticos}

Para el análisis estadístico se utilizó la prueba de Student Newman Keuls como prueba de comparaciones múltiples. En todos los casos el nivel de significación fue del $5 \%$.

\section{Resultados}

Se realizaron tablas y gráficos a modo de descripción de los valores tomados. A los fines de observar la frecuencia del mínimo espesor del elemento dentario, discriminado por zona y cara, se realizaron tablas de contingencia. (Tabla1)

Para analizar los valores medios del espesor residual, se realizó un modelo lineal general mixto, utilizando Zona y Cara como factores y teniendo en cuenta los valores tomados del mismo diente. (Figura 2)

Se usó prueba de Student Newman Keuls como prueba de comparaciones múltiples. En todos los casos el nivel de significación fue del $5 \%$.

Tabla 1. Media, desvío estándar, (DE), mínimo y máximo espesor dentinario, expresado en milímetros discriminados por zona y cara.

\begin{tabular}{|l|c|r|r|r|r|}
\hline Zona & Cara & \multicolumn{1}{|c|}{ Media } & D.E. & Mínimo & Máximo \\
\hline \multirow{2}{*}{$\mathrm{AP}$} & $\mathrm{M}$ & 1,305 & 0,521 & 0,7 & 3,1 \\
\cline { 2 - 6 } $\mathrm{AP}$ & $\mathrm{D}$ & 1,345 & 0,343 & 0,8 & 2,2 \\
\cline { 2 - 6 } $\mathrm{AP}$ & $\mathrm{V}$ & 2,025 & 0,401 & 1,6 & 3,4 \\
\cline { 2 - 6 } $\mathrm{AP}$ & $\mathrm{P}$ & 2,240 & 0,366 & 1,7 & 2,9 \\
\hline $\mathrm{M}$ & $\mathrm{M}$ & 1,515 & 0,394 & 1,1 & 2,6 \\
\cline { 2 - 6 } $\mathrm{M}$ & $\mathrm{D}$ & 1,650 & 0,314 & 1,2 & 2,3 \\
\cline { 2 - 6 } $\mathrm{M}$ & $\mathrm{V}$ & 2,265 & 0,334 & 1,8 & 3,2 \\
\cline { 2 - 6 } $\mathrm{M}$ & $\mathrm{P}$ & 2,495 & 0,341 & 1,9 & 3,2 \\
\hline $\mathrm{C}$ & $\mathrm{M}$ & 1,725 & 0,438 & 0,9 & 2,9 \\
\cline { 2 - 6 } $\mathrm{C}$ & $\mathrm{D}$ & 1,770 & 0,513 & 0,8 & 2,8 \\
\cline { 2 - 6 } $\mathrm{C}$ & $\mathrm{V}$ & 2,355 & 0,398 & 1,8 & 3,2 \\
\cline { 2 - 6 } $\mathrm{C}$ & $\mathrm{P}$ & 2,340 & 0,338 & 1,8 & 2,9 \\
\hline
\end{tabular}

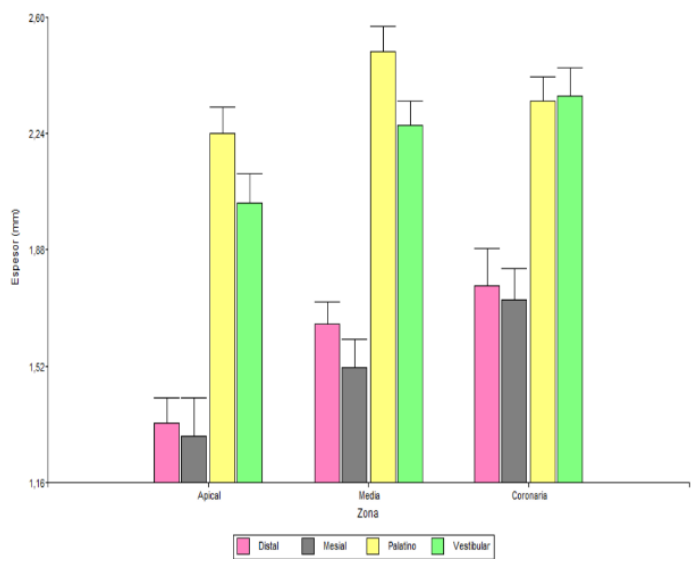

Figura 2. Valores medios y error estándar del espesor dentinario expresado en mm; en los 3 niveles de la raíz (coronario, medio y apical) y en las caras libres y proximales. 


\section{Discusión}

El ensanchamiento del tercio coronario del conducto radicular es de suma importancia ya que permite un mejor acceso al tercio apical del mismo, para eliminación de debris, ensanchamiento del conducto, irrigación y obturación. En algunos casos, los dientes tratados endodónticamente requieren posteriormente la colocación de un poste intraradicular. Estos procedimientos, se traducen a una reducción significativa del espesor de la pared de dentina, debilitando el diente y aumentando las posibilidades de perforaciones $y$ fracturas verticales ${ }^{27}$. El potencial de fractura en dientes tratados endodonticamente aumenta proporcionalmente a la cantidad de dentina removida, siendo de mayor riesgo aquellas raíces de forma oval ${ }^{9}$, como las estudiadas en el presente estudio.

La preparación para poste no solo debilita el diente, sino que también disminuye la capacidad del diente de soportar estrés masticatorio ${ }^{28}$. Es por todo esto que la preservación de dentina es de suma importancia. Al menos $1 \mathrm{~mm}$ de espesor dentinario debe estar presente hacia todos los lados y a lo largo de toda la raíz ${ }^{16}$.

Alexander Katz y Silvia Wasenstein-Kohn ${ }^{29}$ estudiaron este espesor utilizando cortes de los elementos dentarios colocados sobre papel milimetrado y se tomaron fotografías a partir de las cuales se realizaron mediciones a tres alturas de la raíz y hacia ocho lados en cada corte. En la actualidad, los avances en la tecnología nos permitieron realizar este estudio y tomar las mediciones en base a tomografías computarizadas, mejorando la exactitud de las mismas. Estas, fueron tomadas en tres cortes de la raíz (Punto coronario: hombro de tallado coronario, Punto gutapercha: altura del conducto donde comienza la obturación con gutapercha y Punto medio: altura equidistante entre el punto coronario y el punto gutapercha. Y hacia cuatro lados (mesial, distal, vestibular y palatino).

A lo largo del tiempo, se propusieron múltiples protocolos de desobturación del sistema de conductos radiculares, como el descripto en el estudio realizado por Raphael Pilo y Aviad Tamse en el año $2000^{10}$, quienes realizaron la desobturación de los $6 \mathrm{~mm}$ coronales de conducto con fresas Gates Glidden $\mathrm{N}^{\circ} 2$ y posteriormente $\mathrm{N}^{\circ} 4$ y taladros del sistema Para Post $\mathrm{N}^{\circ} 3,4$ y 5. Difiriendo en algunos instrumentos del protocolo realizado en este trabajo: Fresa Gates Glidden $n^{\circ} 2$ a $1000 \mathrm{rpm}$, Fresa PEESO n ${ }^{\circ} 1$ y 2 a $1000 \mathrm{rpm}$ y Ensanchador o "taladro" específico de la marca de postes RTD N², color rojo, 90.06. Con cada uno de los instrumentos se trabajó hasta una longitud pre establecida de acuerdo a la longitud del conducto, dejando $5 \mathrm{~mm}$ de obturación con gutapercha a nivel apical.

Por su parte, Xu Chen $(2019)^{30}$, realizó la medición del espesor de dentina residual utilizando microtomografía computada luego de la colocación virtual del poste de fibra de vidrio. Se realizaron microtomografía de los elementos dentarios seleccionados, como así también de postes de distinto grosor. Posteriormente, con un software (MeVisLab) se colocó digitalmente los postes en el centro del conducto radicular y trazando cuatro líneas en sentido mesial, distal, vestibualr y lingual; en tres cortes a 1,3 y $5 \mathrm{~mm}$ del orificio de entrada de los conductos radiculares, se tomaron las medidas del espesor de dentina residual.

En cuanto a los resultados del presente estudio, en cinco elementos dentarios de los 20 que compusieron la muestra, se encontraron espesores dentinarios menores a $1 \mathrm{~mm}$. En zonas apical y media, la cara con los mínimos más pequeños fue la mesial, mientras que en zona coronal fue la distal. Raphael Pilo en su estudio realizado en el año $2000^{10}$ en premolares inferiores obtuvo resultados similares, observando los menores espesores en los lados mesial y distal, como así también en el nivel más apical.

Por otro lado, Erick Miranda Souza $(2010)^{31}$ obtuvo resultados diferentes en su estudio del espesor de dentina residual en molares superiores en donde se observaron los mínimos espesores en los lados palatino y vestibular, llegando estos incluso a espesores por debajo de 1 $\mathrm{mm}$.

\section{Conclusión}

1. En 5 elementos dentarios el espesor fue menor a $1 \mathrm{~mm}$

2. En zonas apical y media, la cara con los mínimos espesores fue la mesial, mientras que en zona coronal fue la distal.

3. El promedio del espesor de zona apical fue significativamente menor al resto.

4. El promedio de mesial y distal fue significativamente menor al resto.

5. El promedio de los valores de los mínimos espesores se presentaron en las caras mesial y distal de la zona apical, seguido por mesial de la zona media.

De acuerdo a los resultados del presente estudio y basado en la comparación con otros similares, podemos concluir que la preparación del conducto para la colocación de postes radiculares es una práctica riesgosa para las raíces y que debe ser llevada a cabo con cautela. Teniendo en cuenta no sólo la anatomía de los elementos dentarios, ya que 
los puntos más débiles de menor espesor varían de un elemento dentario a otro; sino también un completo conocimiento de los protocolos y diámetros de cada instrumento y sistema de preparación de conductos.

\section{Bibliografía}

1. Morgano SM, Rodríguez AH, Sabrosa CE. Et al. Restoration of endodontically treated teeth. Dent Clín of North América. 2004; 48:397416

2. Guzy GE, Nicholas JI. In vitro comparison of intact endodontically treated teeth with and without endo-post reinforcement. J of Prosth Dent. 1979; 42: 39-44

3. Tamse A. Et al. Iatrogenic vertical root fracturé in endodontically treated teeth. Endod Dent traumatol. 1988; 4:190-6

4. Cheung W. Et al. A review of the mangment of endodontically treated teeth Post, core and the final restoration. Journal of the american dental associaton.2005; 136: 611-9

5. Bertoldi Hepburn A. Et al. Nuevos enfoques en la rehabilitación coronaria del diente endodónticamente tratado. Rev Asoc Odontol Argent.2002; 90:266-75

6. Cheung W. A review of the managment of endodontically treated teeth. Post core and the final restoration. J Am Dent Assoc. 2005;136: 611-9

7. Bertoldi Hepburn A, Ensinas P. Postes radiculares de base orgánica. Ventajas Y limitaciones. Rev Asoc Odontol Argen. 2005;93(1):65-73

8. Bertoldi Hepburn A, Ensinas P. ¿deben los postes de base or gánica reforzados con fibras fijarse en forma adhesiva? Rev Asoc Odontol Argen. 2011; 99: 125-136

9. Pilo R, Shapenco E, Lewinstein I. Residualdentin thickness in bifurcated maxillary first premolars after root canal preparation with parallel-sided drills. J Prosthet Dent. 2008; 99:267-73

10. Pilo R, Tamse A. Residual dentin thickness in mandibular premolares prepeared with Gates glidden and para Post drills. J of Prosth Dent. 2000; 83:617-623

11. Belluci C, Perrini N. A study on the thickness of radicular dentine and cementum in anterior some premolar teeth. Int Endod J. 2008; 35:594-606
12. Cleghorn BM, Christie WH, Dong CC. The root and root canal morphology of the human mandibular second premolar: a literature review. J Endod 2007;33: 1031-7

13. Cohen S, \& Hargreaves KM. Vías de la pulpa. $9^{a}$ ed. Madrid: Elsevier Mosby; 2008. (UDC: RK351 P37)

14. Standlee JP, Caputo AA. Endodontic dowel retention with resinous cements. J Prosthet Dent. 1992; 68:913-7

15. Jhonson JK, Schwartz NL, Blackwell RT. Evaluation and restoration of endodontically treated posterior teeth. J Am Dent Assoc 1976; 93:597-605

16. Caputo AA, Standlee JP. Pins and posts, why, when and how. Dent Clin North Am. 1976; 20:299-311

17. Raiden GC. Gendelman H. Effect of dowel space preparación on the apical seal of root canal fillings. Endod Dent Traumatol. .(1994)

18. Ozyurek, T. Cyclic fatigue resistance of Reciproc, Wave One, and Wave One gold nickel-titanium instruments. $\mathbf{J}$ of Endod. 2016;42 (10), 1536-1539.

19. Ozyurek, T., Uslu, G., Yilmaz, K., \& Gundogar, M. Effect of glide path creating on cyclic fatigue resistance of Reciproc and Reciprocblue nickel-titanium files: A laboratory study. J of Endo. 2018; 44(6), 1033-1037.

20. Koch, A. T. A., Binus, S. M., Holzschuh, B., Petschelt, A., Powers, J. M., \& Berthold. Restoration of endodontically treated teeth with major hard tissue loss - influence of post surface design on pull-out bond strength of fiber-reinforced composite posts. Den Traumatol. 2014; 30(4), 270-279.

21. Souza EM, Bretas RT, Cenci MS, et al. Periapicales radiographs overestimate root canal wall thickness during Post space preparación. Int Endod J. 2008; 41:658-63.

22. Lofthag-Hansen S, Huumonen S, et Al. Limited cone bean CT and intraoral radiography for the diagnosis of periapical pathology. Oral Radiol Endod.2007;103:1149

23. De Paula - Silva FWG, Wu M-K. Leonardo M, et al. Accuracy of periapical radiography and cone bean computed tomography scans in diagnosing apical periodontitis using histopathological findings as gold standard. J Endod 2009; 35:1009-12. 
24. Sukovic P. Cone beam computed tomography in craniofacial imaging. 2003; 1:31.

25. Scarfe WC, Farman AG, Sukovic P. Clinical aplications of cone beam computed tomography in dental practice. J Can Det Assoc. 2006; 72:75.

26. Sathorn C, Palamara JE, Palamara JD, Messer HH. Effect of root canal size and external root surface morphology on fracture susceptibility and pattern: a finite element analysis., J Endod. 2005; 31:288-92

27. Tamse A, Fuss Z, Lustig J, Kaplavi J. An evaluation of endodontically treated vertically fractured teeth. J Endod 1999; 25: 506-8.

28. Trope M, Ray HL, Resistance to fracture in endodontically treated roots. Oral Srg, Oral Med, Oral Pathol 1992; 73:99-102.

29. Katz A, WAsentein-kohn S, Tamse A. Residual dentin thickness in bifurcated maxillary premolars after root canal and dowel space preparation. JOE 2006; 32:202205

30. Chen $\mathrm{X}^{1}$, Min $\mathrm{Y}^{2}$, Gao $\mathrm{Y}^{3}$, Peng $\mathrm{L}$ Microcomputer tomography measurement of minimum residual dentin thickness in mandibular first molars after virtual fiber post placement. J Prosthet Dent 2019 18;1-8.

31. Souza EM, Nascimento LM, Filho M. The impact of post preparation on the residual dentin thickness of maxillary molars. J Posteth Dent 2011 106; 3, 185-191.

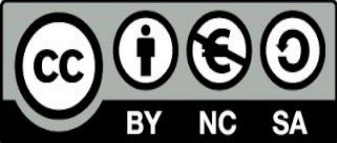

